\title{
Determining Cosmological Parameters from the Microwave Background
}

\author{
Arthur Kosowsky \\ Harvard-Smithsonian Center for Astrophysics, 60 Garden Street, Cambridge, \\ Massachusetts 02138 \\ Marc Kamionkowski \\ Department of Physics, Columbia University, New York, New York 10027 \\ Gerard Jungman \\ Department of Physics, Syracuse University, Syracuse, New York 13244 \\ David N. Spergel \\ Department of Astrophysical Sciences, Princeton University, Princeton, New Jersey 08544 \\ and \\ Department of Astronomy, University of Maryland, College Park, Maryland 20742
}

(May 1996)

\begin{abstract}
Recently funded satellites will map the cosmic microwave background radiation with unprecedented sensitivities and angular resolutions. Assuming only primordial adiabatic scalar and tensor perturbations, we evaluate how accurately experiments of this type will measure the basic cosmological parameters $\Omega$ (the total density of the Universe), $\Omega_{b}$ (the baryon density), $h$ (the Hubble constant), and $\Lambda$ (the cosmological constant). The proposed experiments are capable of measuring these parameters at the few-percent level. We briefly discuss the generality of these estimates and complications arising in actual data analysis.
\end{abstract}

To appear in Proceedings of the UCLA Dark Matter '96 Conference. 
The slight temperature fluctuations in the cosmic microwave background (CMB), first detected by the COBE satellite in 1992 [1], contain a wealth of information about the early Universe [2, 3]. By measuring the power spectrum of these anisotropies, we can hope to extract information about the gross features of the Universe: the Hubble constant $H_{0}=$ $100 h \mathrm{~km} \mathrm{~s}^{-1} \mathrm{Mpc}^{-1}$, which gives the expansion rate; the total energy density $\Omega$ in units of the critical density $\rho_{c}=3 H_{0}^{2} / 8 \pi G$, which determines the geometry of the Universe; the cosmological constant $\Lambda$, the energy density of empty space; and the baryon density $\Omega_{b}$ in terms of the critical density. While information on each of these parameters may be obtained from astronomical measurements, all are notoriously difficult to determine; current estimates are not very precise and are dominated by systematic errors. The microwave background promises a completely independent method of determining all of these parameters. This article largely summarizes the conclusions of previously published work [4,5] to which we refer the reader for details and more extensive references.

Here we estimate the precision with which these cosmological parameters will be determined by a high-resolution, high-sensitivity map of the microwave sky such as that produced by the MAP satellite of NASA [6] (slated for launch in 2000) or ESA's COBRAS/SAMBA mission [7] (planned for 2004). The microwave sky is a statistical realization of an underlying cosmological theory which predicts the temperature power spectrum:

$$
\begin{aligned}
C(\theta) & \equiv\left\langle\frac{\Delta T\left(\hat{\mathbf{q}}_{1}\right)}{T_{0}} \frac{\Delta T\left(\hat{\mathbf{q}}_{2}\right)}{T_{0}}\right\rangle \\
& \equiv \sum_{l=2}^{\infty} \frac{2 l+1}{4 \pi} C_{l} P_{l}(\cos \theta),
\end{aligned}
$$

where $\Delta T(\hat{\mathbf{q}}) / T_{0}$ is the fractional temperature fluctuation in the direction $\hat{\mathbf{q}}, \hat{\mathbf{q}}_{1} \cdot \hat{\mathbf{q}}_{2}=\cos \theta$, $P_{l}$ are Legendre polynomials, and the brackets are an ensemble average over all observers; the mean CMB temperature is $T_{0}=2.726 \pm 0.010 \mathrm{~K}$ [8]. A particular CMB measurement will give an estimate for the values of the multipole moments $C_{l}$. A simple model for a full-sky mapping experiment which treats noise in each pixel as gaussian and neglects correlations between pixels gives an estimated standard error in measuring each $C_{l}$ as 9

$$
\sigma_{l}=\left(\frac{2}{2 l+1}\right)^{1 / 2}\left[C_{l}+w^{-1} \exp \left(l^{2} \sigma_{b}^{2}\right)\right]
$$

where $\sigma_{b}=7.42 \times 10^{-3}\left(\theta_{\mathrm{fwhm}} / 1^{\circ}\right)$ for a gaussian beam, and the inverse weight per solid angle $w^{-1} \equiv\left(\sigma_{\text {pix }} \theta_{\text {fwhm }} / T_{0}\right)^{2}$ is a pixel-size-independent measure of experimental noise. At small $l$ (large angles), the error estimate Eq. 2 is dominated by the first "cosmic variance" term, while at large $l$ (small angles) the noise increases exponentially due to the beam width.

A given cosmological theory will predict the $C_{l}$ values. In the following analysis, we consider the broad class of theories in which the primordial perturbations were adiabatic with roughly a power-law spectrum. This includes all models based on inflation and encompasses many currently popular models such as cold dark matter, mixed dark matter, open models, and $\Lambda$-models. Outside of this class are isocurvature models, including defect models like cosmic strings and textures; some comments on distinguishing between these two classes of models are included below. The adiabatic models are described by the following set of parameters: $\Omega, h, \Lambda$, and $\Omega_{b} h^{2}$, described above; the amplitudes and power-law indices of 
the initial scalar and tensor perturbation spectra, $Q, r \equiv Q_{T} / Q_{S}, n_{S}$, and $n_{T}$, along with another parameter $\alpha \equiv d n_{S} / d \ln k$ which describes the deviation of the scalar spectrum from a perfect power law; the effective number of light-neutrino species at decoupling, $N_{\nu}$; and the total optical depth through the epoch of reionization, $\tau$. Given a set of values $\mathbf{s}$ for these eleven cosmological parameters, we calculate the moments $C_{l}(\mathbf{s})$ using a semi-analytic technique [10,5. The power spectrum can also be calculated by numerically evolving the relevant Boltzmann equations; an efficient and public code for this purpose has been provided in Ref. [11].

Now it is straightforward to determine the precision to which these parameters may be measured given the measurement error estimate Eq. 2. If the Universe is described by the underlying parameter set $\mathbf{s}_{0}$, the probability distribution for observing a CMB power spectrum best fit by the parameter set $\mathbf{s}$ is

$$
P(\mathbf{s}) \propto \exp \left[-\frac{1}{2}\left(\mathbf{s}-\mathbf{s}_{0}\right) \cdot[\alpha] \cdot\left(\mathbf{s}-\mathbf{s}_{0}\right)\right]
$$

where the curvature matrix $[\alpha]$ is given approximately by

$$
\alpha_{i j}=\sum_{l} \frac{1}{\sigma_{l}^{2}}\left[\frac{\partial C_{l}}{\partial s_{i}} \frac{\partial C_{l}}{\partial s_{j}}\right]_{\mathrm{s}=\mathrm{s}_{0}} .
$$

In statistical terminology, the matrix $[\alpha]$ is known as the Fisher information matrix [12]. The inverse of this matrix, the covariance matrix $[\mathcal{C}]=[\alpha]^{-1}$, gives estimates for the uncertainties in measuring the parameters: when all parameters are fit simultaneously, the variance in $s_{i}$ is $\mathcal{C}_{i i}$. If some of the parameters are fixed by other means, the variances on the rest are given by inverting the appropriate submatrix of $[\alpha]$.

In Fig. 1, we display the standard errors for the parameters $\Omega, \Lambda, h$, and $\Omega_{b} h^{2}$ given an underlying "standard CDM" model defined by the parameters $\Omega=1, h=0.5, \Omega_{b} h^{2}=0.01$, $\Lambda=0,3$ light neutrinos, no reionization, no tensor perturbations, and a flat initial power spectrum of scalar perturbations normalized to the COBE quadrupole, $Q=18 \mu \mathrm{K}$ [13]. Displayed as a function of beam size are the standard ("1- $\sigma$ ") errors obtainable from a fullsky mapping experiment with two different noise levels $w^{-1}=4.2 \times 10^{-15}$ and $1.3 \times 10^{-17}$. The first weight corresponds to the $90 \mathrm{GHz}$ channel of MAP while the second corresponds to the $143 \mathrm{GHz}$ channel of COBRAS/SAMBA. The large disparity in sensitivity is due to the difference between HEMT and bolometer technology: bolometers attain substantially better sensitivity but require active cooling to $\mathrm{mK}$ temperatures. In these frequency channels, MAP will have a nominal angular resolution of $0.29^{\circ}$ and COBRAS/SAMBA a resolution of $0.17^{\circ}$. Note these error estimates assume no information about any of the parameters, i.e. an 11-parameter fit to the model. Our analysis assumes no systematic errors (e.g. in foreground removal, beam profile measurement, calculation of $C_{l}$ 's) which lead to systematic misestimates of the cosmological parameters.

The CMB thus in principle offers the possibility of measuring the basic cosmological parameters with far better precision than traditional astronomical techniques can offer. A natural question is the generality of these results. We have assumed a particular cosmological model and a highly idealized experiment; what happens when these assumptions are relaxed?

We have calculated the expected variances for several different underlying cosmological models with similar results, unless the universe is substantially open or has undergone 

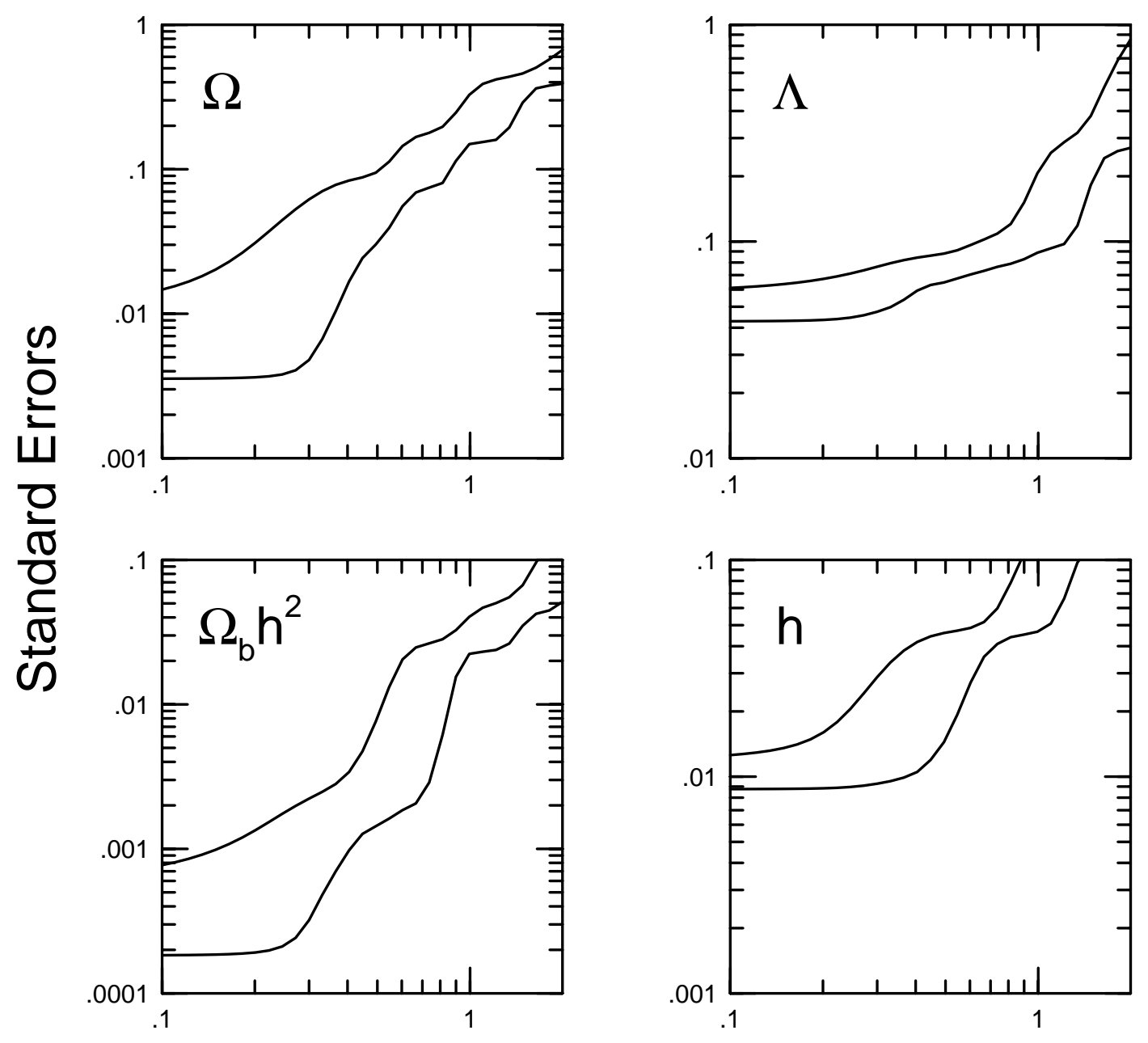

Beamwidth in degrees

FIG. 1. Standard errors from a full-sky mapping experiment as a function of beam width for noise levels $w^{-1}=4.2 \times 10^{-15}$ (upper curve) and $1.3 \times 10^{-17}$ (lower curve). The underlying model is "standard CDM."

significant reionization. In the first case, features in the power spectrum are shifted to smaller angular scales, weakening the parameter determination for a given beam size. However, this displacement of power spectrum features is a robust signature of an open universe which is difficult for any other cosmological model to mimic [14, 15], so the geometry of the universe will still be determined to high precision. In the second case, if the total optical depth back to the last scattering epoch is of order unity, features in the power spectrum will be greatly reduced in amplitude, hindering parameter determination. Current degree-scale anisotropy detections from a variety of ground and balloon experiments make this possibility unlikely 16,17.

Structure formation may not have resulted from initial adiabatic perturbations outside of the horizon, as with the inflation-type models we have considered, but rather from isocurvature fluctuations in some type of defect model (i.e. cosmic strings or textures). We do not yet possess highly accurate calculations of the CMB power spectrum in such models, 
but recent work suggests that the power spectrum will generically possess a substantially different structure than in the adiabatic case [15, 18]. If the Universe is actually described by cosmic strings or textures, a microwave background map should provide an unambiguous signature, although the extent to which cosmological parameters could also be determined remains an open question.

The ultimate barrier to extracting the information in the CMB is foreground sources. At microwave frequencies, galactic synchrotron, free-free, and dust emission are can be of comparable amplitude to the CMB temperature fluctuations [19,20]; in the region of the galactic plane, the foregrounds swamp the anisotropy signal. Additionally, at angular scales below 10' radio point sources may be a serious problem. The galactic plane will be cut from any CMB map as COBE did; statistical techniques for analyzing the resulting partial-sky map are well-known [21]. For the rest of the sky, the foreground signals can be removed because they possess frequency dependences much different from blackbody. MAP will measure in five frequency bands ranging from 22 to $90 \mathrm{GHz}$, while COBRAS/SAMBA will measure in nine channels from 30 to $900 \mathrm{GHz}$; these frequency spreads should be sufficient for foreground separation at a high level of accuracy.

Finally, all of our calculations of theoretical CMB power spectra are performed in linear perturbation theory. At scales below a half degree, various non-linear physical effects begin to contribute at a non-negligible level: gravitational lensing of the microwave background by large-scale structure [22]; the Sunyaev-Zeldovich effect from hot clusters [23]; and the ReesSciama effect from non-linear cluster evolution [24]. While these effects generally only give corrections to the $C_{l}$ 's of a few percent, the errors induced by neglecting them are systematic. Any analysis of a high-resolution map, particularly at angular scales below 10', should include all of these effects for accurate parameter determination. The estimates here are performed by truncating the sum in Eq. (4) at $l=1000$. The COBRAS/SAMBA experiment can in principle make use of information at much smaller scales than this: the lower curves in the figure become flat below quarter-degree resolution because the measurement becomes dominated by cosmic variance out to $l=1000$. If theoretical models are understood well enough, if foregrounds are not a serious problem, and if the beam is determined well enough to allow probing scales substantially smaller than the beam size, the error estimates presented here for COBRAS/SAMBA at the smallest angular scales could be surpassed.

In conclusion, upcoming experiments to map the cosmic microwave background at high sensitivity and angular resolution promise very exciting results. If the Universe is described by an inflation-type model with a near power-law spectrum of initial adiabatic perturbations, such a map will provide us with precise determination of the basic cosmological parameters $\Omega$, $\Lambda, h$, and $\Omega_{b}$. If on the other hand the Universe is described by a defect model ore some other unanticipated possibility, these experiments will likely indicate this unambiguously. Either way, the next decade should bring a great increase in our knowledge of the fundamental properties of the Universe. 


\section{ACKNOWLEDGMENTS}

This work was supported in part by the D.O.E. under contracts DEFG02-92-ER 40699 at Columbia University and DEFG02-85-ER 40231 at Syracuse University, by the Harvard Society of Fellows, by the NSF under contract ASC 93-18185 (GC3 collaboration) at Princeton University, by NASA under contract NAG5-3091 at Columbia University and NAGW-2448 and under the MAP Mission Concept Study Proposal at Princeton University. 


\section{REFERENCES}

[1] G. F. Smoot et al., Astrophys. J. Lett. 396 (1992), L1.

[2] J.R. Bond, in Cosmology and Large-Scale Structure (Proceedings of Les Houches School, 1993), ed. R. Schaefer, Elsevier, Amsterdam, 1995.

[3] W. Hu, N. Sugiyama, and J. Silk, Nature in press (1996), astro-ph/9604166.

[4] G. Jungman, M. Kamionkowski,

A. Kosowsky, and D.N. Spergel, Phys. Rev. Lett. 76 (1996) 1007.

[5] G. Jungman, M. Kamionkowski,

A. Kosowsky, and D.N. Spergel, Phys. Rev. D in press (1996), astro-ph/9512139.

[6] See the MAP home page at http://map.gsfc.nasa.gov.

[7] See the COBRAS/SAMBA home page at http://astro.estec.esa.nl:80/SA-general/Projects/Cobras/cobras.htm].

[8] J. C. Mather et al., Astrophys. J. Lett. 420 (1994) 439.

[9] L. Knox, Phys. Rev. D 52 (1995) 4307.

[10] W. Hu and N. Sugiyama, Phys. Rev. D 51 (1995) 2599; Astrophys. J. 444 (1995) 489.

[11] U. Seljak and M. Zaldarriaga, Astrophys. J. submitted (1996), astro-ph/9603033.

[12] M. Tegmark, A.N. Taylor, and A.F. Heavens, Mon. Not. R. Ast. Soc. submitted (1996), astro-ph/9603021.

[13] C.L. Bennett et al., Astrophys. J. 464, L1 (1996).

[14] M. Kamionkowski, D.N. Spergel, and

N. Sugiyama, Astrophys. J. Lett. 426 (1994) L57.

[15] W. Hu and M. White, Astrophys. J. submitted (1996), astro-ph/9602019.

[16] M. White, D. Scott, and J. Silk, Ann. Rev. Astron. Astrophys. 32 (1994) 329.

[17] C.B. Netterfield et al., Astrophys. J. submitted (1996), astro-ph/9601197.

[18] J. Magueijo et al., Phys. Rev. Lett. 76 (1996) 2617.

[19] M. Tegmark and G. Efstathiou, Mon. Not. R. Ast. Soc. in press (1996), astroph/9507099.

[20] S. Dodelson, Astrophys. J. submitted (1996), astro-ph/9512021.

[21] K. Gorski, Astrophys. J. 430 (1994) 85.

[22] U. Seljak, Astrophys. J. 463 (1996) 1.

[23] R. Sunyaev and Ya. B. Zeldovich, Ann. Rev. Astron. Astrophys. 18 (1980) 537.

[24] M. J. Rees and D. W. Sciama, Nature 517 (1968) 611. 